\title{
Research on Library Information Consultation Model on Cloud Computing Platform
}

\author{
Hongmin Wang \\ College of Information Science and Technology, Bohai University, Jinzhou, 121013, China \\ wanghongmin0704@163.com
}

Keywords: cloud computing; digital library; virtual reference; library management

\begin{abstract}
The rapid development of computer and network technology, the advent of the era of cloud quietly, more and more profound influence and change people's lives. The cloud computing give human life a new force, but also for the social life human beings a whole new trading patterns. The emergence of cloud computing also indicates that the emergence of an unprecedented Information technology and business computing model. In the new form of the Internet, library information and consulting services to gradually shift from the traditional to the information service model in the new network environment. The technology and concept of cloud computing applications in library services, will inevitably affect the library services technology, ideas, ways and means. Combining with the status quo of the current library information consulting services, to create the stage cloud computing platform information consulting services for the feasibility analysis, put forward the construction scheme of library information consulting service in the cloud platform and architecture design.
\end{abstract}

\section{Introduction}

The existing information consulting service model is not only high in input expenses, lacks efficiency in controlling but also repetitive and monotonous in labor. With the emergency, development and being applied in the library information consulting service. The technology of the cloud computing reduces some repetitive labor, improve service quality and efficiency, reinforce the reliability of the service contents and decrease the cost. The more important is the cloud computing has very large-scale data storage capacity and high speed calculation ability, which makes the existing library consulting service model obtained radical change[1].

\section{Library Information Consulting Service Status Analyses}

The information consulting service of traditional libraries is a kind of service ways which helps consumers and readers solve the problem of how to obtain collection resource. With the constant progress of technology, the content of information referential and consulting service also represents the tendency of deep-going development: turn the service of facing the masses gradually to personalized subject service. The traditional consulting service analyzes and arranges the related words and data mainly according to the reader's and user's demands in data information resources, thus getting information source of more connotations by secondary operation. Its essence is carrying out readers' and users' demand analysis firstly, the reclassification to the approach and origin of the related information and integrating (assembling) the types of information fit for the demands of the readers and the users.

With the extension of the Internet, traditional information consulting services also derive a new way of digital reference service. The services refer to the ways of information obtaining by which readers and users consult some questions online directly and industry experts offer the answers through the application of computers and network. Its essence is still the traditional consulting service.

With the development of search engine technology, the users' way of accessing data is varying quietly. Some big search engine websites like Baidu, Google and so on have already become the main way of accessing data information for users. "Google Answers" pushed out by Google , " 
Baidu knows" pushed out by Baidu, "iask" pushed out by Sina and other information consulting services by internets marked the beginning of era of computer information consulting services and divided information consulting service into traditional information consulting service and network information consulting service, which both can be mutually complementary.

At the present stage, we list the research characteristics of the cloud computing applied in the library consulting service area as follows:

(1) Development of research is very fast, but not profound enough. Some new research force are added formally, but related theoretical studies remain some limitations, the introductions are on preliminary stage and essence and instances are much less.

(2) The scope of study is wide, but there's no planning and arrangement wholly. Later advantages are more obvious. In fact, it is in 2009 that our country began actually to apply cloud computing technology into the area of library consulting service. The content of research is from the concept of cloud computing gradually to all related service area in library and then into all levels of information consulting, such as: information and data security, blog and mobile phone library and resource sharing.

\section{Advantages Analysis}

Cloud computing technology has the characteristics of which data has amount of storage, wide rang and strong ability of calculating ,if leading it into the counseling of library, it can change the primary method of calculation and then change the information service way for readers subvert the library's traditional ideas of living on simple information access and counseling.

In a larger extent, realizes information integration and resource sharing. Join "cloud computing" information service mode, data stored in the cloud, selected by the user to quickly extract to information,Library together around the store data on the same server, to build a "data cloud". In the "cloud" mode, the readers don't need to purchase a server or software software resources, as long as through the PC connect to the network, a network share can be through the cloud computing to find the required information resources, virtual computer environm ent, etc. Of course users to enjoy cloud services shall be paid correspondingly.

Service level: Being service providers, libraries give cloud service providers data and information beyond bag form. Cloud service providers use virtualization and network technology, according to the centering on the needs of user' service mode, making the service industry of library trend to be multi-level and rounded. As the user of information, readers can surf the internet to set information from the "cloud" by the facilities like computers and mobile phones whenever and wherever possible [2, 3].

And in a cloud environment, not only on the user's computer configuration is low, timely server maintenance is mainly composed of cloud computing terminal provider, library all kinds of maintenance is greatly reduced.

Concept level: change the direction of library construction. First at all, cloud computing using the information resources, sharing way solves the prices for books and periodicals, lead to new problems of the decrease in the number of hidden bibliography. Second, cloud computing technology can statistics out some sort of document resources utilization, is advantageous for the library in understanding the current degree of readers' demand, rich resources and improve the books. Third, cloud computing has increased the reader using the frequency and amount of network literature resources, make the deeper and more diverse forms of data.

Cloud computing services will really change the library literature collection mode, there will be original form into a single collection of cloudy way, at the same time also increased the utilization rate of books.

A cloud environment of the library, the strong participatory cloud computing information resource scarcity caused by hardware will weaken the influence of convenience; readers will pay more attention to software services. 


\section{Construct the Scheme of Cloud Computing Library Information Consulting Service}

4.1 The library building need to be aware of problems in cloud computing environment

Cloud model is the core of all kinds of cloud technology, it is the basic model of qualitative quantitative transformation. From the perspective of the general situation of cloud computing application, the application of cloud computing is still in the primary stage of commercial application, there are many, including network performance, security, loss, standard use of problems, such as these are plagued by "cloud" service providers and users. At Gartner released a report called the cloud computing security risk assessment is pointed out that the cloud of the risk of seven in the privileged user access, to review, data, data isolation, data recovery, survey support, from the point of the current situation, main need to cause the attention of the library has the following several aspects:

(1) the standard problem

Due to the construction of the library under the influence of various factors, such as the imperfection of IT infrastructure, economic capability and library itself could not update IT infrastructure, often also can't completely abandoned the existing equipment, but in the current mode and based on the existing equipment, such as deployment pattern and the cloud computing model for effective integration, updating of the existing equipment, materials and data, and so on. Due to the different cloud service businesses in different technical standards, resulting in different companies system cannot be used to match, the difference of the library infrastructure, poor compatibility, access speed difference, thus greatly limits the data exchange and sharing of information between different libraries. In April 2009, standardization organization "the distributed management task force" to form a "development of cloud computing standard incubator", by Microsoft, Internet, many well-known large companies such as IBM's strong support, visible cloud computing company also hopes to achieve reunification of the standard and technology integration.

(2) data security problem

Data is a powerful library resources guarantee, some unpredictable factors from the outside world, such as network hacker intrusion, etc., will cause a loss of library data resources. The resources of the library collection mainly include the form of digital resources and some resources with independent property rights. Libraries can deposit the copyrighted resources to the cloud, in the process of actual operation, there may be some unexpected reasons led to the suspension of the server, so the library needs to set up a self synchronization backup device, data recovery and backup in time to prevent accidental situation; To prevent the accident occurs, the library can normal work. For the other libraries, they have their own core data, so all of the data sources are stored in the cloud is not possible, need to a sort of data, in the local establish a corresponding backup data packets to store self-built and important data, ensure the security of data. Not only guarantee the security of the data, but also can solve the current library will backup all data is stored or the data stored in the local damage caused by the data and problems such as insufficient memory [4].

(3) the intellectual property and data confidentiality problem

In cloud services, data and resources in a cloud, therefore the existence of the data confidentiality and privacy protection issues. This requires matching appropriate digital security rules and protocols, formulate corresponding resource usage and scope, to ensure data security, safeguard the rights and interests of authors, to ensure the security of data and personal privacy.

(4) the cost problem

Through the operation of cloud computing in the library, the library can be of some important hardware devices and daemon to provide cloud services, thus can reduce the equipment upgrade, the cost of maintenance, etc. On the other hand, also save the corresponding manpower resource and energy consumption, etc. However, when we put all the resources and the daemons are running in the cloud, excessive dependence on centralized control may lead to cloud computing products in one supplier, service and market monopoly. Therefore need to improve the guarantee mechanism of the market. On the other hand, the library can be based on the hardware and software of the differences and the server and need to choose a different service, transfer between service providers can also cause cost increase. Therefore, in the process, database developers, software developers 
and the need for mutual cooperation between the library and developed to meet user needs on the basis of standardized protocol and requirements of interface software, so as to safeguard the rights and interests of the library.

(5) consciousness shift

The operation mode of the traditional library will gradually shift by modern thinking, consciousness of librarians for guidance, make its use the reader's demand and new cloud model.

4.2 Cloud computing is a kind of fusion power.

With the help of network storage technology, distributed processing technology and the virtualization technology to data integration classification. In order to achieve a new type of technical advisory service. In the cloud, the data is stored in the background of the "cloud" service. Users only need to have a network device. You can get the relevant data whenever and wherever possible information and services. For cloud users do not care about the "cloud" as it provides the computer services. But pay more attention to the final data which is a unified interface after processing.

The dominant direction of digital library construction is the network infrastructure. Taking information community as its service object, digital library lays emphasize on creating virtual space, makes use of cloud computing to support community through the most advanced information technology, transferring and shifting technology to make the readers and users have access to all kinds of information so as to give the users a totally-new experience [5,6].

4.3 The architecture design of library information consulting service under the cloud computing environment

Under the cloud environment, the library is not only the technological supporter of the cloud technology, but also the realization site supporters of cloud services. In the process of the integration of cloud computing and library, according to the relationship between the two, we divide the library construction into three forms: the related cloud computing built by the library itself; cloud computing rented partly; cloud computing rented entirely. The relationship mode of library and cloud service is shown as Fig.1.

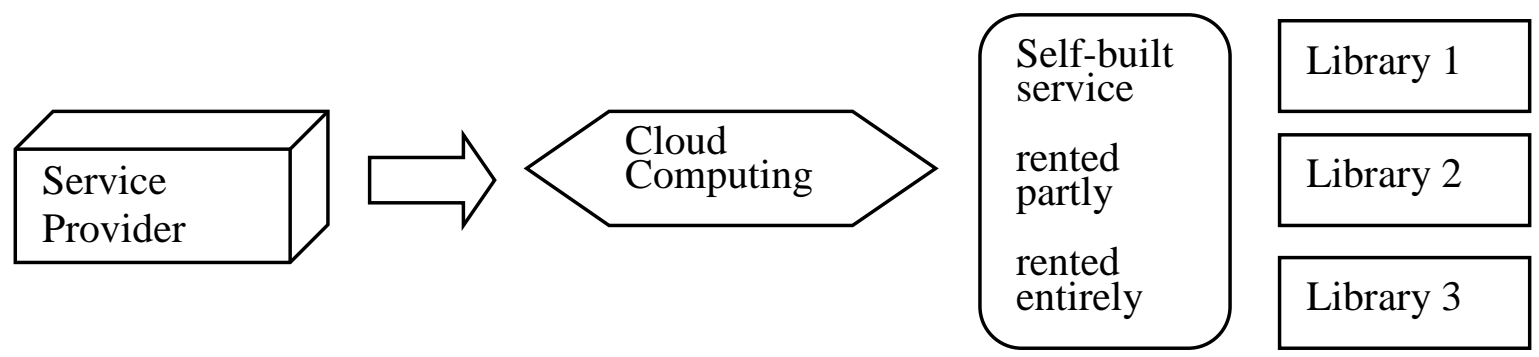

Fig.1. the relationship between library and cloud service

(1) Self-service library development to create a cloud service. On April 23, 2009, OCLC launched "web collaborative library management and service", has been generally accepted as a specific service, its purpose is to replace the existing each kind of library integrated management system [7]. Library is only a specific link in a cloud environment, it will be the museum information resources stored in the cloud data terminal, readers and users through the network to realize data sharing and the background of utilization of resources, so as to improve operation efficiency.

(2) Library part of cloud services. Library will be some of the more important data stored in self-built service system and send those who are not very important resources exist in the cloud, like a common data information and operation information in the library itself, etc can be stored in the cloud on the server[8,9], save cost and improve the efficiency of the library. Because of the virus and network some unsafe factors caused by emergencies is inevitable, so it cannot ensure that data information completely in the library itself can be controlled within the scope of the library can create your own data management system to manage the data information resources. 
(3) Rented entirely library cloud computing service. Under this kind of service of the library, without independent research and development and maintenance of the system, you can access and obtain the data needed information resources as long as provide a fee for cloud service providers. By filtering and selecting the cloud data information, the library chooses the data meeting the demands of readers and users and at the same time saves the expenses and time of research, development and maintenance of the system, thus allowing the library to commit energy to the other affairs [10].

\section{Conclusion}

In short, the development of the library experienced a shift from the traditional era to the digital environment. It experienced from the network era, web 2.0era and as well as the grid era. Library reference service is also slowly toward the era of cloud development path, and cloud computing as a new technology, still need continue to practice and perfect. The level of the concept of "cloud" computing technology, ideas, service mode, step-by-step guide future development path of the library and scale, especially in the digital library virtual reference services, this will be the future impact of domestic and foreign books the idea of a major museum field, through the planning of a new development concept and decision-making in the library constraints cloud computing will become an important infrastructure development of the library, as well as some policy programs and regulations, the books Hall and cloud services will be better together. These will guide us how to properly use cloud computing, advanced digital technology to improve library service skills, mode, scale and enhance the efficiency of library services to improve library services.

\section{Acknowledgment}

This work is supported by social science fund project of Liaoning province (L13DTQ015).

\section{References}

[1] G. Bai, "Applied Study of Cloud Computing in the Library Information Consultation Service," Liaoning Normal University, pp. 8-22, 2013.

[2] X. J. Hu, B. S. Fan, "The Challenges of Library Management Taken by Cloud computing," University Library Journal, vol. 30, no.4, pp.7-12, 2009.

[3] Y. Zhang, "The Application Model Research of Digital Library Based on Cloud Computing," Computer Programming Skills and Maintenance, vol. 20, no. 10, pp.47-48, 2013.

[4] X. Yu, "Research on the Cloud Services Platform Architecture of Digital Library Based on Cloud Computing Technology," Information Science, vol. 29, no. 7, pp. 1049-1053, 2011.

[5] F. J. Kong. "The Influence and Thought of Cloud Computing on Libraries," Library and Information, vol. 28, no.2, pp. 93-95, 2010.

[6] Duncan J.Watts. "Six Degrees: The Science of a Connected Age," W.W.Norton \& Company, vol. 31, no.2, pp.384, 2004.

[7] H. Chai, J. Yan, Q. Y. Qin, "The model of fuzzy evaluation of software quality based on confidence," Computer engineering and design, vol. 33, no. 2, pp. 607-611, 2012.

[8] R. H. Wang, "Improvement of personal credit evaluation model of support vector machine based on consumer credit," Statistics and Decision, vol. 26, no. 11, pp. 54-56, 2010.

[9] J. F. Su, "The project management of the software development process quality, "Heilongjiang Science and Technology Information, vol. 13, no. 2, pp. 118-119, 2009.

[10] X. S. Wu, "Discussion on software quality assurance of software development process, "Study of Science and Engineering at RTVU, vol. 32, no. 1, pp. 48-50, 2010. 